\title{
Trial Primary Objective
}

National Cancer Institute

\section{Source}

National Cancer Institute. Trial Primary Objective. NCI Thesaurus. Code C85826.

The principal purpose of the trial. 